\title{
Lipoprotein(a) - Einfluss auf die kardiovaskuläre Manifestation
}

\author{
K.-P. Mellwig · C. Schatton • B. Biermann • \\ T. Kottmann $\cdot$ D. Horstkotte $\cdot$ F. van Buuren
}

Online publiziert: 10. Februar 2015

(C) Die Autor(en) 2015. Dieser Artikel ist auf Springerlink.com mit Open Access verfügbar

Zusammenfassung Der klinischen Relevanz von Lipoprotein(a) [Lp(a)] als kardiovaskulärer Risikofaktor wird aktuell nur bedingt die notwendige Bedeutung beigemessen. Es gilt zu klären, welchen Einfluss erhöhte Lp(a)-Werte auf die Entstehung und den Schweregrad einer koronaren Herzkrankheit (KHK) hat.

In einer retrospektiven Analyse erfolgte die Auswertung von 31.274 Patienten, die erstmals stationär aufgenommen wurden. Es wurden Patienten verglichen mit isoliert erhöhtem $\mathrm{Lp}(\mathrm{a})(>110 \mathrm{mg} / \mathrm{dl})$ und normwertigen $\mathrm{Lp}(\mathrm{a})(<30 \mathrm{mg} /$ dl), mit steigenden Lp(a)-Konzentrationen (30-60 mg/dl, 61-90 mg/dl, 91-110 mg/dl) und in einer dritten Analyse mit zusätzlich erhöhtem LDL-Cholesterin und HBA1c.

Die Patienten mit hohem Lp(a) wiesen im Vergleich zu denen mit normwertigem Lp(a) signifikant häufiger eine fortgeschrittene KHK auf bei einem Anteil der Dreigefäßerkrankungen von 50,2 vs. $25,1 \%$, hatten anamnestisch signifikant häufiger einen Myokardinfarkt (34,6 vs.16,6\%; $p<0,001)$, eine operative Myokardrevaskularisation $(40,8$ vs. $20,8 \% ; p<0,001)$ und eine Koronarintervention $(55,3$ vs. $33,6 \% ; p<0,001)$ durchgemacht. Auffällig ist ein deutlicher Geschlechtsunterschied zu Ungunsten der männlichen Patienten in der Ausbildung und des Schweregrades der KHK. Das Risiko für eine KHK (Odds Ratio) war bei $\mathrm{Lp}(\mathrm{a}) \geq 110 \mathrm{mg} / \mathrm{dl}$ um das 5,5-fache erhöht. Bei Patienten mit zusätzlich erhöhtem LDL und $\mathrm{HbA1c}$ war keine zu-

Dr. med. K.-P. Mellwig $(\bowtie) \cdot$ C. Schatton · B. Biermann

T. Kottmann $\cdot$ D. Horstkotte $\cdot$ F. van Buuren

Klinik für Kardiologie, Herz- und Diabeteszentrum NRW,

Universitätsklinik der Ruhr-Universität Bochum,

Georgstr. 11,

32545 Bad Oeynhausen, Deutschland

E-Mail: kpmellwig@hdz-nrw.de sätzliche Erhöhung der KHK-Häufigkeit und Anstieg des Schweregrades zu verzeichnen.

Mit steigender Lp(a)-Konzentration nehmen Manifestation und Schweregrad der koronaren Herzkrankheit zu. Zusätzliche Risikofaktoren führen zu keiner Agravierung in der Manifestation der koronaren Herzkrankheit.

Schlüsselwörter Lipoprotein(a) · Koronare

Herzkrankheit · LDL-Cholesterin · HbA1c

\section{Lipoprotein(a): influence on cardiovascular manifestation}

Abstract The clinical relevance of lipoprotein(a) (Lp(a)) as a cardiovascular risk factor is currently underestimated. The aim of our study was to assess the influence of increased $L p(a)$ values on the development and severity of coronary artery disease (CAD).

In our retrospective analysis of 31,274 patients, who were hospitalized for the first time, we compared patients with isolated increased $\mathrm{Lp}(\mathrm{a})(>110 \mathrm{mg} / \mathrm{dl})$ and normal $\mathrm{Lp}$ (a) $(<30 \mathrm{mg} / \mathrm{dl})$, with increased $\mathrm{Lp}(\mathrm{a})$ concentrations (30-60 mg/dl, 61-90 mg/dl, 91-110 mg/dl), and in a third analysis with additionally increased LDL cholesterol and HbA1c values.

Patients with high $L p(a)$ levels showed a significantly higher incidence of advanced CAD with a three-vessel disease being present in 50.2 vs. $25.1 \%$. Patients with high $\mathrm{Lp}$ (a) levels had a significantly more frequent history of myocardial infarction ( 34.6 vs. $16.6 \%, p<0.001)$, surgical myocardial revascularization $(40.8$ vs. $20.8 \%, p<0.001)$ and percutaneous coronary intervention (55.3 vs. $33.6 \%$, $p<0.001)$. In addition, there was a marked difference in gender to the disadvantage of male patients regarding de- 
velopment and severity of CAD. CAD risk (Odds ratio) was increased 5.5 -fold in patients with $\mathrm{Lp}(\mathrm{a}) \geq 110 \mathrm{mg} / \mathrm{dl}$. Additionally elevated LDL and HbA1c levels were not associated with increased manifestation and severity of CAD.

High Lp(a) concentration leads to an increased manifestation and severity of coronary artery disease. Additional risk factors do not aggravate manifestation of CAD.

Keywords Lipoprotein(a) C Coronary artery disease · LDL cholesterol $\cdot \mathrm{HbA} 1 \mathrm{c}$

\section{Einleitung}

Lp(a) - erstmals 1963 von Kare Berg beschrieben - gehört zur Klasse der cholesterinreichen, apo-B-haltigen Lipoproteine des menschlichen Plasmas [2]. Es besteht aus einem Low-Density-Lipoprotein (LDL)-Partikel und dem Lp(a)spezifischen Glykoprotein Apolipoprotein-a [Apo(a)]. Die Struktur des Apo(a) setzt sich aus Aminosäuresequenzen zusammen, die als „Kringels“ bezeichnet werden. Die Struktur ist genetisch festgelegt und unterscheidet sich in Ihren Isoformen in der Häufigkeit des Kringel-IV-Typ 2. Apo(a) ist in seiner Struktur zu 90\% homolog zu Plasminogen, hat jedoch keine fibrinolytische Wirkung wie Plasminogen, Blutgerinnsel aufzulösen. $L p(a)$ hat somit atherogene Eigenschaften wie LDL und thrombogene Eigenschaften durch die Homogenität zu Plasminogen.

Die Bedeutung von $\mathrm{Lp}$ (a) als Risikofaktor für eine koronare Herzkrankheit wird kontrovers diskutiert [7, 33]. Es liegen nur wenige Daten über die Auswirkung auf den Koronarstatus und kardiale Ereignisse bei einer isolierten exzessiven Lp(a)-Erhöhung vor [20].

In einer retrospektiven Analyse durch Auswertung von Patientendaten des Herz- und Diabeteszentrum NRW sollte Folgendes geklärt werden:

a. Gibt es bei isolierter, exzessiver Lp(a)-Erhöhung Geschlechtsunterschiede in der Manifestation der koronaren Herzkrankheit?

b. Lässt sich ein Schwellenwert bei steigender Lp(a)Konzentration in der Manifestation der koronaren Herzkrankheit nachweisen?

c. Wie wirken sich zusätzliche Risikofaktoren auf Manifestation der koronaren Herzkrankheit aus?

\section{Patienten und Methoden}

Retrospektiv wurden die Datensätze von 31.274 Patienten ausgewertet, die erstmalig im Herz- und Diabeteszentrum NRW aufgenommen wurden.
In einer ersten Analyse wurden Patienten mit einem $\mathrm{HbA} 1 \mathrm{c} \leq 6,1 \%$, einem LDL $<130 \mathrm{mg} / \mathrm{dl}$ sowie bekanntem Koronarstatus ausgewählt. Die insgesamt 265 Patienten mit einer Lp(a)-Konzentration $\geq 110 \mathrm{mg} / \mathrm{dl}$ wurden nach Matching bezüglich Alter und Geschlecht mit einer Gruppe aus 319 Patienten mit einem $L p(a)<30 \mathrm{mg} / \mathrm{dl}$ verglichen.

In einer zweiten Analyse wurden Patienten mit zunehmender Lp(a)-Konzentration sowie einem $\mathrm{HbAlc} \leq 6,1 \%$, einem LDL $<130 \mathrm{mg} / \mathrm{dl}$ und koronarangiographisch dokumentiertem Koronarstatus ausgewählt (s. Tab. 1). Insgesamt kamen in den unterschiedlichen Gruppen 2119 Patienten zur Auswertung.

In einer dritten Analyse sollte die Auswirkung zusätzlich bestehender Risikofaktoren wie erhöhtes LDL-Cholesterin und eine diabetische Stoffwechsellage (erhöhtes HbA1c) auf die Manifestation der koronaren Herzkrankheit untersucht werden (s. Tab. 2). Aus dem Gesamtkollektiv wurden 953 Patienten (603 männliche, 350 weibliche) in vier Stoffwechselgruppen statistisch ausgewertet.

Die statistischen Auswertungen erfolgten mit SPSS für Windows, Version 19.0 (SPSS Inc., USA). Die metrischen Variablen wurden als Mittelwerte/Standardabweichungen dargestellt. Bei allen durchgeführten Tests wurde eine zweiseitige Signifikanzüberprüfung vorgenommen, wobei für alle statistischen Verfahren ein $p$-Wert $<0,05$ als statistisch signifikant angenommen wurde.

\section{Ergebnisse}

Patienten mit hohem $L p(a)$ wiesen signifikant häufiger eine fortgeschrittene KHK auf mit einem Anteil an Dreigefäßerkrankung (3-GE) von 50,2 vs. $25,1 \%$. Das Risiko für eine KHK (OR) war bei $\mathrm{Lp}(\mathrm{a}) \geq 110 \mathrm{mg} / \mathrm{dl}$ um das 5,5fache erhöht. Bei der Analyse der Schwere der KHK wiesen Männer deutlich unterschiedliche Befunde im Vergleich zu den untersuchten Frauen auf. Eine Eingefäßerkrankung (1-GE) wurde nachgewiesen bei $11,7 \%(n=21 / 179)$ der Männer und 18,6\% $(n=16 / 86)$ der Frauen. Zweigefäßerkrankungen (2-GE) waren bei 19,4\% $(n=35 / 179)$ der Männer und 16,2\% $(n=14 / 86)$ der Frauen nachweisbar. 58,3\% ( $n=105 / 179)$ der Männer wiesen eine Dreigefäßerkrankung (3-GE) auf, im Gegensatz waren nur 33,3\% $(n=29 / 86)$ Frauen betroffen.

Das Alter bei den Patienten mit erhöhten Lp(a)-Konzentrationen war bei Erstmanifestation der KHK im Mittel um ca. 5 Jahre signifikant niedriger als in der Kontrollgruppe (Median: 61,9 vs. 66,6 Jahre).

Patienten mit hohem Lp(a) hatten anamnestisch signifikant häufiger einen Myokardinfarkt (34,6 vs.16,6\%; $p<0,001)$, eine operative Myokardrevaskularisation $(40,8$ vs. $20,8 \% ; p<0,001)$ und eine Koronarintervention $(55,3$ vs $33,6 \% ; p<0,001)$ durchgemacht. 
Tab. 1 Analyse von Patienten mit zunehmender Lp(a)-Konzentration

\begin{tabular}{|c|c|c|c|c|c|c|}
\hline \multicolumn{2}{|c|}{ Lp(a)-Gruppe (mg/dl) } & \multicolumn{4}{|l|}{ KHK } & \multirow[t]{2}{*}{ Gesamt } \\
\hline & & Nein & 1-GE & 2-GE & $3-\mathrm{GE}$ & \\
\hline \multirow[t]{2}{*}{$\leq 5,0$} & Anzahl & 225 & 26 & 22 & 26 & 299 \\
\hline & Innerhalb der Lp(a)-Gruppe (\%) & 75,3 & 8,7 & 7,4 & 8,7 & 100,0 \\
\hline \multirow[t]{2}{*}{$5,1-10$} & Anzahl & 77 & 9 & 6 & 8 & 100 \\
\hline & Innerhalb der Lp(a)-Gruppe (\%) & 77,0 & 9,0 & 6,0 & 8,0 & 100,0 \\
\hline \multirow[t]{2}{*}{$10,1-15$} & Anzahl & 99 & 18 & 14 & 24 & 155 \\
\hline & Innerhalb von $L p(a)$-Gruppe (\%) & 63,9 & 11,6 & 9,0 & 15,5 & 100,0 \\
\hline \multirow[t]{2}{*}{$15,1-20$} & Anzahl & 38 & 9 & 8 & 10 & 65 \\
\hline & Innerhalb der Lp(a)-Gruppe (\%) & 58,5 & 13,8 & 12,3 & 15,4 & 100,0 \\
\hline \multirow[t]{2}{*}{$20,1-25$} & Anzahl & 104 & 34 & 27 & 50 & 215 \\
\hline & Innerhalb der Lp(a)-Gruppe (\%) & 48,4 & 15,8 & 12,6 & 23,3 & 100,0 \\
\hline \multirow[t]{2}{*}{$25,1-30$} & Anzahl & 73 & 22 & 18 & 44 & 157 \\
\hline & Innerhalb der Lp(a)-Gruppe (\%) & 46,5 & 14,0 & 11,5 & 28,0 & 100,0 \\
\hline \multirow[t]{2}{*}{$30,1-40$} & Anzahl & 96 & 30 & 22 & 56 & 204 \\
\hline & Innerhalb der Lp(a)-Gruppe (\%) & 47,1 & 14,7 & 10,8 & 27,5 & 100,0 \\
\hline \multirow[t]{2}{*}{$40,1-50$} & Anzahl & 71 & 20 & 11 & 38 & 140 \\
\hline & Innerhalb der Lp(a)-Gruppe (\%) & 50,7 & 14,3 & 7,9 & 27,1 & 100,0 \\
\hline \multirow[t]{2}{*}{$50,1-60$} & Anzahl & 56 & 14 & 22 & 43 & 135 \\
\hline & Innerhalb der Lp(a)-Gruppe (\%) & 41,5 & 10,4 & 16,3 & 31,9 & 100,0 \\
\hline \multirow[t]{2}{*}{$60,1-90$} & Anzahl & 44 & 51 & 39 & 90 & 224 \\
\hline & Innerhalb der Lp(a)-Gruppe (\%) & 19,6 & 22,8 & 17,4 & 40,2 & 100,0 \\
\hline \multirow[t]{2}{*}{$90,1-110$} & Anzahl & 35 & 32 & 32 & 62 & 161 \\
\hline & Innerhalb der Lp(a)-Gruppe (\%) & 21,7 & 19,9 & 19,9 & 38,5 & 100,0 \\
\hline \multirow[t]{2}{*}{$>110$} & Anzahl & 46 & 37 & 48 & 133 & 264 \\
\hline & Innerhalb der Lp(a)-Gruppe (\%) & 17,4 & 14,0 & 18,2 & 50,4 & 100,0 \\
\hline Gesamt & Anzahl & 964 & 302 & 269 & 584 & 2119 \\
\hline
\end{tabular}

Tab. 2 Untersuchung der Auswirkung zusätzlicher Risikofaktoren

\begin{tabular}{|c|c|c|c|c|c|}
\hline \multirow[t]{2}{*}{ KHK } & & \multicolumn{4}{|l|}{ Stoffwechselgruppe } \\
\hline & & $\begin{array}{l}\text { Lp(a) niedrig LDL } \\
\text { und HbA1c niedrig }\end{array}$ & $\begin{array}{l}\text { Lp(a) hoch, LDL und } \\
\text { HbAlc niedrig }\end{array}$ & $\begin{array}{l}\text { Lp(a) hoch, LDL } \\
\text { hoch, HbA1c niedrig }\end{array}$ & $\begin{array}{l}\text { Lp(a), LDL und } \\
\text { HbAlc hoch }\end{array}$ \\
\hline \multirow[t]{2}{*}{ Nein } & Anzahl & 150 & 45 & 80 & 19 \\
\hline & Anteil von Stoffwechselgr (\%) & 47,0 & 17,0 & 28,7 & 21,3 \\
\hline \multirow[t]{2}{*}{$1 \mathrm{GE}$} & Anzahl & 50 & 37 & 50 & 17 \\
\hline & Anteil von Stoffwechselgr (\%) & 15,7 & 14,0 & 17,9 & 19,1 \\
\hline \multirow[t]{2}{*}{$2 \mathrm{GE}$} & Anzahl & 39 & 49 & 48 & 8 \\
\hline & Anteil von Stoffwechselgr (\%) & 12,2 & 18,5 & 17,2 & 9,0 \\
\hline \multirow[t]{2}{*}{$3 \mathrm{GE}$} & Anzahl & 80 & 134 & 101 & 45 \\
\hline & Anteil von Stoffwechselgr (\%) & 25,1 & 50,6 & 36,2 & 50,6 \\
\hline
\end{tabular}

In einer zweiten Analyse wurden die Daten von 2119 Patienten mit steigender Lp(a)-Konzentration untersucht (Tab. 1).

Auffällig ist, dass schon bei einer Lp(a)-Konzentration zwischen 25,1-30 mg/dl, dem oberen Normwert für Lp(a), $53,5 \%$ der Untersuchten (14,0\% 1-GE, 11,5\% 2-GE, $28 \%$ 3-GE) eine KHK aufwiesen; bei einer Lp(a)-Konzentration $>110 \mathrm{mg} / \mathrm{dl}$ wurde in $82,6 \%$ der Fälle eine KHK nachgewiesen (14,0\% 1-GE, 18,2\% 2-GE, 50,4\% 3-GE). Mit steigender Lp(a)-Konzentration zeigte sich eine zunehmende Häufung der Dreigefäßerkrankung (Abb. 1).

In der dritten Analyse sollte der Einfluss zusätzlich bestehender Risikofaktoren beurteilt werden.
Insgesamt wurden aus dem Gesamtkollektiv unter Berücksichtigung der Einschlusskriterien 953 Patienten ausgewählt. Die Gruppeneinteilung unter Berücksichtigung von $\mathrm{Lp}(\mathrm{a})$, LDL und dem HbAlc wurde in vier Stoffwechselgruppen vorgenommen:

Stoffwechselgruppen:

0: Lp(a), LDL, und HbAlc normwertig $(n=319)$

1: $\mathrm{Lp}(\mathrm{a}) \geq 110 \mathrm{mg} / \mathrm{dl}$, LDL und HbA1c normwertig $(n=265)$

2: $\mathrm{Lp}(\mathrm{a}) \geq 110 \mathrm{mg} / \mathrm{dl}, \mathrm{LDL}>150 \mathrm{mg} / \mathrm{dl}, \mathrm{HbA} 1 \mathrm{c}$ normwer$\operatorname{tig}(n=280)$

3: $\mathrm{Lp}(\mathrm{a}) \geq 110 \mathrm{mg} / \mathrm{dl}, \mathrm{LDL}>150 \mathrm{mg} / \mathrm{dl}, \mathrm{HbAlc}>6,1 \%$ $(n=89)$ 


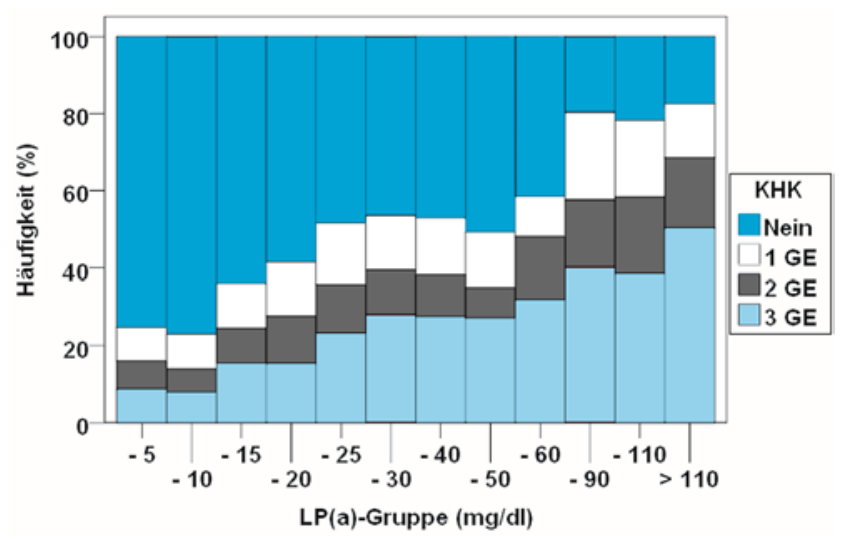

Abb. 1 Koronarstatus bei den verschiedenen Lp(a)-Gruppen

In Stoffwechselgruppe 0 mit niedrigem Lp(a) wiesen 25,1\% $(n=80)$ der Patienten eine 3-GE auf. Im Patientenkollektiv der Stoffwechselgruppe 1 mit erhöhtem Lp(a) und niedrigem LDL und HbA1c wurden mit 50,6\% $(n=134)$ doppelt so viele Patienten mit einer 3-GE diagnostiziert, während in Stoffwechselgruppe 2 und 3 mit zusätzlich erhöhtem LDL bzw. HbA1c kein weiterer Anstieg der 3-GE zu verzeichnen war (Tab. 2).

\section{Diskussion}

Ein $\mathrm{Lp}(\mathrm{a})>30 \mathrm{mg} / \mathrm{dl}$ gilt allgemein als pathologisch, wobei über die Bedeutung exzessiv erhöhter Werte wenig bekannt ist $[6,8,13,25,26,27,41]$. Sehr hohe Lp(a)-Konzentrationen über $100 \mathrm{mg} / \mathrm{dl}$ sind sehr selten [16, 21] zur Auswertung gekommen.

Zahlreiche Studien belegen [3, 5, 13, 17-19, 24, 28, 36, 40-42, 45, 46], dass hohe Lp(a)-Plasmakonzentrationen als Risikofaktor für kardiovaskuläre Erkrankungen zu werten sind. Über die Ausprägung der KHK bei erhöhtem Lp(a) liegen nur wenige Daten vor [47].

Die Auswertung der in der ersten Analyse ermittelten Daten zeigte eine signifikant höhere Inzidenz für eine 3-GE bei den Patienten mit hohen Lp(a)-Serumspiegeln (52,0 vs $25,2 \%)$.

In der Zusammenschau zeigt die geschlechtsdifferente Untersuchung, dass Männer häufiger und schwerer in Abhängigkeit von der Höhe des Lp(a)-Spiegels an KHK erkrankten, dass aber auch bei Frauen ein hoher Lp(a)Spiegel mit erhöhten Risiken für KHK einhergeht.

Untersucht man die Literatur hinsichtlich des Alters der Patienten bei Bekanntwerden der KHK, so ist festzustellen, dass die Manifestation der KHK mit steigenden Lp(a)Spiegeln vornehmlich jüngere Populationen betrifft $[4,8$, 9-12, 22, 29, 32, 39, 44].

Anhand der Daten konnte gezeigt werden, dass das Alter bei den Patienten mit erhöhten Lp(a)-Konzentrationen bei
Erstmanifestation der KHK im Mittel um ca. fünf Jahre signifikant niedriger war als in der Vergleichsgruppe.

Bei der Frage nach einem Schwellenwert für das Auftreten einer KHK wird in der Literatur auf den stufenweisen Anstieg des Risikos für einen Myokardinfarkt bei ansteigender Lp(a)-Konzentration hingewiesen [20]. Ein Schwellenwert konnte nicht ermittelt werden. Keine Angaben liegen vor über den Schweregrad der KHK bei ansteigender Lp(a)-Konzentration.

In dem untersuchten Patientenkollektiv lag bei noch normwertiger Lp(a)-Konzentration die Inzidenz der KHK bei über $53,5 \%$. Mit steigender Lp(a)-Konzentration ist sowohl ein Anstieg der KHK-Manifestation (Lp(a) $>110 \mathrm{mg} / \mathrm{dl}:$ 80,2\% KHK) als auch eine Zunahme des Schweregrades (3-GE 50,4\%) feststellbar. Ein eindeutiger Schwellenwert war nicht festzustellen (Abb. 1).

In der Literatur wird ein modulierender Effekt von Lp(a) auf das KHK-Risiko diskutiert [1, 31, 32, 35, 36, 38, 39], wobei einige Autoren einen additiven Effekt von Risikofaktoren und erhöhtem Lp(a) beschreiben [15, 43].

Im vorliegenden Kollektiv wiesen Patienten aus Stoffwechselgruppe 0 mit $53 \%$ den geringsten Anteil mit einer KHK-Manifestation auf. In der Stoffwechselgruppe 1 bei Patienten mit isoliert erhöhtem Lp(a) nahm die Rate der KHK im Vergleich zur Gruppe ohne dokumentierte Stoffwechselstörungen signifikant zu. Bei der genauen Einteilung der koronaren Herzerkrankungen in 1-, 2- und 3-GE stach hervor, dass in Stoffwechselgruppe 1 die 3-GE mit 50,6\% doppelt so häufig auftrat wie in Stoffwechselgruppe 0. Die Rate an Patienten mit einer 3-GE stieg weder bei zusätzlich erhöhtem LDL noch bei erhöhtem LDL und HbA1c, wie erwartet, nicht weiter an.

\section{Zusammenfassung}

In dem untersuchten Patientenkollektiv lässt sich eindeutig ein Geschlechtsunterschied zu Ungunsten der männlichen Patienten in Form einer erhöhten Inzidenz der koronaren Herzkrankheit, insbesondere in der Ausbildung einer Mehrgefäßerkrankung bei steigender Lp(a)-Konzentration nachweisen, assoziiert mit einer höheren Rate an Myokardinfarkten, Koronarinterventionen und einer früheren Erstmanifestation von kardialen Ereignissen.

Schon bei einer Lp(a)-Konzentration zwischen 25,1 und $30,0 \mathrm{mg} / \mathrm{dl}$ wies das untersuchte Patientenkollektiv in über $50 \%$ der Fälle eine KHK mit überwiegender Ausbildung einer 3-GE auf. Ab einer Lp(a)-Konzentration $>90 \mathrm{mg} / \mathrm{dl}$ stieg die Rate auf über $80 \%$ an.

Zusätzliche Risikofaktoren wie erhöhtes LDL-Cholesterin und eine diabetische Stoffwechsellage scheinen in dem untersuchten Kollektiv keinen Einfluss auf die Ausbildung und den Schweregrad der koronaren Herzkrankheit zu haben. 
Die erhobenen Daten belegen eindrucksvoll, dass ein erhöhtes Lp(a) als bedeutsamer Risikofaktor für eine koronare Herzkrankheit zu werten ist. Bei der Erstellung des kardiovaskulären Risikoprofils sollte dieser Faktor mit erfasst werden. Bei einer Lp(a)-Konzentration $>100 \mathrm{mg} /$ $\mathrm{dl}$ ist eine weiterführende Diagnostik gerechtfertigt, da mit hoher Wahrscheinlichkeit eine Mehrgefäßerkrankung zu erwarten ist.

Die Lipidapherese ist derzeit bei aktuell fehlender medikamentöser Beeinflussung nachweislich die einzige effektive Therapieoption bei erhöhter Lp(a)-Konzentration [23].

Interessenkonflikt K.-P. Mellwig, C. Schatton, B. Biermann, T. Kottmann, D. Horstkotte und F. van Buuren erklären, dass kein Interessenkonflikt besteht.

Open Access Dieser Artikel unterliegt den Bedingungen der Creative Commons Attribution License. Dadurch sind die Nutzung, Verteilung und Reproduktion erlaubt, sofern der/die Originalautor/en und die Quelle angegeben sind.

\section{Literatur}

1. Ashfaq F, Goel PK, Sethi R, Khan MI, Ali W, Idris MZ (2013) Lipoprotein(a) levels in relation to severity of coronary artery disease in north indian patients. Heart Views 14:12-16. doi:10.4103/1995-705X.107114HV-14-12 [pii]

2. Berg K (1963) A new serum type system in man -the Lp System. Acta Pathol Microbiol Scand 59:369-382

3. Berg K, Dahlen G, Christophersen B, Cook T, Kjekshus J, Pedersen T (1997) Lp(a) lipoprotein level predicts survival and major coronary events in the Scandinavian Simvastatin Survival Study. Clin Genet 52:254-261

4. Bostom AG, Cupples LA, Jenner JL, Ordovas JM, Seman LJ, Wilson PW, Schaefer EJ, Castelli WP (1996) Elevated plasma lipoprotein(a) and coronary heart disease in men aged 55 years and younger. A prospective study. JAMA 276:544-548

5. Buechler C, Ullrich H, Aslanidis C, Bared SM, Lingenhel A, Ritter M, Schmitz G (2003) Lipoprotein (a) downregulates lysosomal acid lipase and induces interleukin-6 in human blood monocytes. Biochim Biophys Acta 1642:25-31 doi:S0167488903000831 [pii]

6. Catalano M, Perilli E, Carzaniga G, Colombo F, Carotta M, Andreoni S (1998) Lp(a) in hypertensive patients. J Hum Hypertens 12:83-89

7. Cremer P, Nagel D, Labrot B, Mann H, Muche R, Elster H, Seidel D (1994) Lipoprotein $\mathrm{Lp}(\mathrm{a})$ as predictor of myocardial infarction in comparison to fibrinogen, LDL cholesterol and other risk factors: results from the prospective Gottingen Risk Incidence and Prevalence Study (GRIPS). Eur J Clin Invest 24:444-453

8. Dahlen GH, Guyton JR, Attar M, Farmer JA, Kautz JA, Gotto AM, $\mathrm{Jr}$ (1986) Association of levels of lipoprotein Lp(a), plasma lipids, and other lipoproteins with coronary artery disease documented by angiography. Circulation 74:758-765

9. von Eckardstein A Schulte H Cullen P Assmann G (2001) Lipoprotein(a) further increases the risk of coronary events in men with high global cardiovascular risk. J Am Coll Cardiol 37:434-439

10. Frohlich J, Dobiasova M, Adler L, Francis M (2004) Gender differences in plasma levels of lipoprotein (a) in patients with angiographically proven coronary artery disease. Physiol Res 53:481-486
11. Gaeta G, Cuomo S, Capozzi G, Foglia MC, Barra S, Madrid A, Stornaiuolo V, Trevisan M (2008) Lipoprotein(a) levels are increased in healthy young subjects with parental history of premature myocardial infarction. Nutr Metab Cardiovasc Dis 18:492-496. doi:S0939-4753(07)00106-8[pii]10.1016/j.numecd.2007.03.006

12. Genest J, Jr, Jenner JL, McNamara JR, Ordovas JM, Silberman SR, Wilson PW, Schaefer EJ (1991) Prevalence of lipoprotein (a) [Lp(a)] excess in coronary artery disease. Am J Cardiol 67:10391145. doi:0002-9149(91)90862-F [pii]

13. Genest JJ, McNamara JR, Salem DN, Schaefer EJ (1991) Prevalence of risk factors in men with premature coronary artery disease. Am J Cardiol 67:1185-1189. doi:0002-9149(91)90924-A [pii]

14. Genest J, Jr, McNamara JR, Ordovas JM, Jenner JL, Silberman SR, Anderson KM, Wilson PW, Salem DN, Schaefer EJ (1992) Lipoprotein cholesterol, apolipoprotein A-I and B and lipoprotein (a) abnormalities in men with premature coronary artery disease. $\mathrm{J}$ Am Coll Cardiol 19:792-802. doi:0735-1097(92)90520-W [pii]

15. Genest JJ, Jr, Martin-Munley SS, McNamara JR, Ordovas JM, Jenner J, Myers RH, Silberman SR, Wilson PW, Salem DN, Schaefer EJ (1992) Familial lipoprotein disorders in patients with premature coronary artery disease. Circulation 85:2025-2033

16. Glader CA, Birgander LS, Stenlund H, Dahlen GH (2002) Is lipoprotein(a) a predictor for survival in patients with established coronary artery disease? Results from a prospective patient cohort study in northern Sweden. J Intern Med 252:27-35. aid:997 [pii]

17. Golden SH, Folsom AR, Coresh J, Sharrett AR, Szklo M, Brancati F (2002) Risk factor groupings related to insulin resistance and their synergistic effects on subclinical atherosclerosis: the atherosclerosis risk in communities study. Diabetes 51:3069-3076

18. Hahmann HW, Schätzer-Klotz D, Bunte T (1999) The significance of high levels of lipoprotein(a) compared with etablished risk factors in premature coronary artery disease. Diffrences between men and woman. Atherosclerosis 144:7

19. Imhof A, Rothenbacher D, Khuseyinova N, Hoffmeister A, Maerz W, Nauck M, Scharnagl H, Koenig W, Brenner H (2003) Plasma lipoprotein Lp(a), markers of haemostasis and inflammation, and risk and severity of coronary heart disease. Eur J Cardiovasc Prev Rehabil 10:362-370. doi:10.1097/01.hjr.0000087080.83314.be

20. Jones GT, van Rij AM, Cole J, Williams MJ, Bateman EH, Marcovina SM, Deng M, McCormick SP (2007) Plasma lipoprotein(a) indicates risk for 4 distinct forms of vascular disease. Clin Chem 53:679-685. doi:clinchem.2006.079947 10.1373/ clinchem.2006.079947[pii]

21. Kamstrup PR, Benn M, Tybjaerg-Hansen A (2008) Extreme lipoprotein(a) levels and risk of myocardial infarction: th Copenhagen City HEART sTUDY. Circulation 8

22. Kamstrup PR, Benn M, Tybjaerg-Hansen A, Nordestgaard BG (2008) Extreme lipoprotein(a) levels and risk of myocardial infarction in the general population: the Copenhagen City Heart Study. Circulation 117:176-184. doi:10.1161/ CIRCULATIONAHA.107.715698

23. Kamstrup PR, Tybjaerg-Hansen A, Steffenssen R (2009) Genetically elevated lipoprotein(a) and increased risk of myocardial infarction. JAMA 301:2331-2339

24. Klausen IC, Sjol A, Hansen PS, Gerdes LU, Moller L, Lemming L, Schroll M, Faergeman O (1997) Apolipoprotein(a) isoforms and coronary heart disease in men: a nested case-control study. Atherosclerosis 132:77-84. doi:S0021-9150(97)00071-3 [pii]

25. Leebmann J, Roeseler E, Julius U, Heigl F, Spitthoever R, Heutling D, Breitenberger P, Maerz W, Lehmacher W, Heibges A, Klingel R (2013) Lipoprotein apheresis in patients with maximally tolerated lipid-lowering therapy, lipoprotein(a)-hyperlipoproteinemia, and progressive cardiovascular disease: prospective observational multicenter study. Circulation 128:2567-2576. doi:10.1161/ circulationaha.113.002432 
26. Luc G, Bard JM, Arveiler D, Ferrieres J, Evans A, Amouyel P, Fruchart JC, Ducimetiere P (2002) Lipoprotein (a) as a predictor of coronary heart disease: the PRIME Study. Atherosclerosis 163:377-384. doi:S0021915002000266 [pii]

27. Mestre C, Grand A, Fournis Y, Mamelle N, Landrault E, Cambrillat N (1998) [The importance of lipoprotein(a) as a predictive factor of coronary atherosclerosis]. Presse Med 27:849-854

28. Murase T, Okubo M, Amemiya-Kudo M, Hiraga T, Oka J, Shimada M, Igarashi T (2007) Impact of markedly elevated serum lipoprotein(a) levels ( $>$ or $=100 \mathrm{mg} / \mathrm{dL}$ ) on the risk of coronary heart disease. Metabolism 56:1187-1191. doi:S00260495(07)00151-5 [pii] 10.1016/j.metabol.2007.04.015

29. Murase T, Okubo M, Amemiya-Kudo M, Ebara T, Mori Y (2008) Impact of elevated serum lipoprotein (a) concentrations on the risk of coronary heart disease in patients with type 2 diabetes mellitus. Metabolism 57:791-795

30. Nicholls SJ, Tang WH, Scoffone H, Brennan DM, Hartiala J, Allayee H, Hazen SL (2010) Lipoprotein(a) levels and long-term cardiovascular risk in the contemporary era of statin therapy. J Lipid Res 51:3055-3061

31. Nordestgaard BG, Chapman MJ, Ray K, Boren J, Andreotti F, Watts GF, Ginsberg H, Amarenco P, Catapano A, Descamps OS, Fisher E, Kovanen PT, Kuivenhoven JA, Lesnik P, Masana L, Reiner Z, Taskinen MR, Tokgozoglu L, Tybjaerg-Hansen A, European Atherosclerosis Society Consensus P (2010) Lipoprotein(a) as a cardiovascular risk factor: current status. Eur Heart J 31:28442853. doi:10.1093/eurheartj/ehq386

32. Onat A, Hergenc G, Ozhan H, Kaya Z, Bulur S, Ayhan E, Can G (2008) Lipoprotein(a) is associated with coronary heart disease independent of metabolic syndrome. Coron Artery Dis 19:125-131

33. Ornek E, Murat S, Duran M, Turfan M, Kurtul A, Demircelik MB, Vatankulu MA, Ocek H, Akdemir R (2011) The relationship between lipoprotein(a) and coronary artery disease, as well as its variable nature following myocardial infarction. Clin Invest Med 34:E14-20

34. Pineda J, Marin F, Marco P, Roldan V, Valencia J, Ruiz-Nodar JM, Sogorb F, Lip GY (2009) Premature coronary artery disease in young $($ age $<45)$ subjects: interactions of lipid profile, thrombophilic and haemostatic markers. Int J Cardiol 136:222-225. doi:S0167-5273(08)00598-6 [pii]10.1016/j.ijcard.2008.04.020

35. Ridker PM, Hennekens CH, Stampfer MJ (1993) A prospective study of lipoprotein(a) and the risk of myocardial infarction. JAMA 270:2195-2199

36. Salomaa V, Rasi V, Pekkanen J, Vahtera E, Jauhiainen M, Vartiainen E, Myllyla G, Ehnholm C (1994) Haemostatic factors and prevalent coronary heart disease; the FINRISK Haemostasis Study. Eur Heart J 15:1293-1299
37. Sawabe M, Tanaka N, Nakahara K, Hamamatsu A, Chida K, Arai T, Harada K, Inamatsu T, Ozawa T, Naka MM, Matsushita S (2009) High lipoprotein(a) level promotes both coronary atherosclerosis and myocardial infarction: a path analysis using a large number of autopsy cases. Heart 95:1997-2002. doi:hrt.2008.160879 [pii]10.1136/hrt.2008.160879

38. Schaefer EJ, Lamon-Fava S, Jenner JL, McNamara JR, Ordovas JM, Davis CE, Abolafia JM, Lippel K, Levy RI (1994) Lipoprotein(a) levels and risk of coronary heart disease in men. The lipid Research Clinics Coronary Primary Prevention Trial. JAMA 271:999-1003

39. Schreiner PJ (1994) Lipoprotein(a) as a risk factor for preclinical atherosclerotic disease in a biracial cohort: the Atherosclerosis Risk in Communities (ARIC) Study. Chem Phys Lipids 67-68:405-410

40. Schwartzman RA, Cox ID, Poloniecki J, Crook R, Seymour CA, Kaski JC (1998) Elevated plasma lipoprotein(a) is associated with coronary artery disease in patients with chronic stable angina pectoris. J Am Coll Cardiol 31:1260-1266

41. Seed M, Ayres KL, Humphries SE, Miller GJ (2001) Lipoprotein (a) as a predictor of myocardial infarction in middle-aged men. Am J Med 110:22-27

42. Seman LJ, DeLuca C, Jenner JL, Cupples LA, McNamara JR, Wilson PW, Castelli WP, Ordovas JM, Schaefer EJ (1999) Lipoprotein(a)-cholesterol and coronary heart disease in the Framingham Heart Study. Clin Chem 45:1039-1046

43. Shai I, Rimm EB, Hankinson SE, Cannuscio C, Curhan G, Manson JE, Rifai N, Stampfer MJ, Ma J (2005) Lipoprotein (a) and coronary heart disease among women: beyond a cholesterol carrier? Eur Heart J 26:1633-1639. doi:ehi222 [pii] 10.1093/eurheartj/ ehi222

44. Shai I, Schulze MB, Manson JE, Stampfer MJ, Rifai N, Hu FB (2005) A prospective study of lipoprotein(a) and risk of coronary heart disease among women with type 2 diabetes. Diabetologia 48:1469-1476. doi:10.1007/s00125-005-1814-3

45. Starkman HS, Cable G, Hala V, Hecht H, Donnelly CM (2003) Delineation of prevalence and risk factors for early coronary artery disease by electron beam computed tomography in young adults with type 1 diabetes. Diabetes Care 26:433-436

46. Thomas HP, Steinhagen-Thiessen E (2003) [Lipoprotein(a): Aspects of pathophysiology, epidemiology and treatment]. Z Kardiol 92:III53-III58

47. Tselepis AD, Elisaf M, Goudevenos J, Tselegaridis T, Bairaktari E, Siamopoulos KC, Sideris D (1996) Lipid profile in patients with microvascular angina. Eur J Clin Invest 26:1150-1155 